\title{
Iterative Soft Multipath Interference Cancellation Assisted by Hybrid ARQ with Constellation rearrangement for HSDPA System
}

\author{
Mohamed Et-tolba \\ and Samir Saoudi \\ Department of Signal \\ and Communications \\ GET - ENST Bretagne \\ Technopôle Brest - Iroise, CS 83818 \\ 29238 Brest Cedex 3, France \\ Email: mohamed.ettolba@enst-bretagne.fr \\ Mahmoud Ammar \\ Sys'Com Laboratory \\ ENIT \\ BP 37, le belvédère 1002 \\ Tunis, Tunisia \\ Email: mahmoud.ammar@enit.rnu.tn
}

\author{
Raphael Visoz \\ France Telecom R\&D \\ DMR/IIM \\ 38-40, rue du générale-Leclerc \\ F-92792 Issy Moulineaux \\ Cedex 9, France
}

Email: raphael.visoz@francetelecom.com

\begin{abstract}
High Speed Downlink Packet Access (HSDPA) is the major evolution of UMTS downlink. It provides paek data rates up to $10.8 \mathrm{Mbps}$. This is reached by radio link adaptivity, which includes fast scheduling, adaptive modulation and coding (AMC), and hybrid automatic reapeat request (HARQ). The performance of AMC suffers from multipath interference introduced by the channel especially when using 16-QAM modulation. This is because of the variation in bit reliabilities caused by the bitmapping onto the signal contellation. In this paper, we present a soft iterative multipath interference canceller (MPIC) for suppressing the multipath interference. We also propose to use HARQ Chase combining with the constellation rearrangement technique in joint application with MPIC. This can equalize the variation of bit reliabilities by using different mapping rules over HARQ transmissions. It is proven that the performance of adaptive modulation and coding is significantly improved after only two HARQ transmissions.
\end{abstract}

\section{INTRODUCTION}

The major evolotion of the UMTS standard in recent year is the high speed downlink packet access (HSDPA). It is designed to provide services at high data rates. The use of multicode transmission principle with adaptive modulation and coding (AMC) as well as hybrid automatic repeat request (HARQ) makes it possible to reach peak data rates up to $10.8 \mathrm{Mbps}$.

In the AMC technique, the base station adapts the modulation and the coding rate to the channel conditions. The coding rates are obtained by the puncturing operation at the output of the turbo code. The modulation is selected between QPSK and 16-QAM. The use of high order modulation (16QAM) increase significantly the bit-rate. However, this type of modulation is very sensitive to interference. This leads to a severe performance degradation in mutlipath fading channel due to the multipath interference. As a solution to mitigate the performance degradation when using 16-QAM modulation is the implementation of a multipath interference canceller (MPIC) at the receiver. Through last years, many studies were carried out on the MPIC. In [3], authors proposed a multipath interference canceller (MPIC) wich generate the multipath interference based on hard-decision results at the output of the Rake receiver. Although the interference rejection weight used in [3] reduces the impact of generation error of the MPI replica, the optimum of this replica cannot be generated. A soft MPIC combined with HARQ were proposed in [4]. It was proven that more accurate replica is genareted. In this paper, we propose an iterative soft MPIC combined with HARQ Chase combining and constellation rearrangement. In our approach, more relible packets are delivered to turbo decoder by changing the signal constellation mapping from transmission to transmission and making soft combining after interference cancellation. This decrease significantly the data-decoding error. The paper is orgonized as follows. In section II, we describe the principle of the iterative soft MPIC. We present the iterative soft MPIC combined with Chase combining and constellation rearrangement in section III. In section IV, we present the simultor configuration. After, simulation results and discussion are given is section $\mathrm{V}$, concluding remarks are made in section VI.

\section{ITERATIVE SOFT MULTIPATH INTERFERENCE CANCELLER}

\section{A. Principle of iterative soft MPIC}

The iterative soft multipath interference canceller used in this work is presented in Fig. 1. The interference is generated using a soft symbol estimation after the SISO demapper.

For multicode DS-CDMA system, the received signal can be written as

$$
r(t)=\sum_{l=1}^{L} g_{l}(t) \sum_{k=1}^{K} s_{k}\left(t-\tau_{l}\right) \cdot c_{k}\left(t-\tau_{l}\right)+n(t)
$$

where $g_{l}$ and $\tau_{l}$ are the complex channel cefficient and the time delay of $l^{\text {th }}$ path, $L$ and $K$ are the number of paths and the number of physical channels respectivelly, $s_{k}$ and $c_{k}$ represent 




Fig. 1. Iterative soft MPIC structure

the modulated signal and spreading code of the $k^{\text {th }}$ physical channel, and $n(t)$ is the complex AWGN noise. In this paper we assumed perfect channel knowledge.

The iterative soft MPIC works in an iterative manner. Its purpose is to eliminate the multipath interference of each Rake finger. For each iteration, the MPIC estimates the multipath interference and substract it from the received signal at the input of each finger which then performs descramling, despreading, and apply the channel conjugate. After that, a maximum ratio combining (MRC) is done for all fingers outputs [5], [8]. The combined sequence is then taken by the SISO demapper to estimate the log-likelihood ratios (LLR) of transmitted bits. The demapper output is delivered to a soft symbols estiamtion function in order to reconstruct the data modulated sequence. This reconstructed sequence is spread, scrambled and processed by a channel emulator which generates all paths interference contributions [6], [7]. This multipath interferences are stored in a memory to be used in succeding iterations.

At $p^{t} h$ stage, the Rake combined sequence of the $n^{\text {th }}$ symbol of the $k^{\text {th }}$ physical channel is writen as

$$
z_{k}^{(p)}(n)=\sum_{l=1}^{L} y_{k, l}^{(p)}(n) \cdot\left(g_{l}^{*}\right)^{(p)}
$$

The estimated multipath interference (MPI) associated with the $l^{\text {th }}$ path at $p^{\text {th }}$ iteration can be expressed as

$$
\hat{I}_{l}^{(p)}\left(t-\tau_{l}\right)=g_{l}^{(p-1)}(t) \cdot \sum_{k=1}^{K} \tilde{s}_{k}^{(p-1)}\left(t-\tau_{l}\right) \cdot c_{k}\left(t-\tau_{l}\right)
$$

The input signal to the $l^{t h}$ finger in the $p^{t h}(p \geq 2)$ iteration is represented as

$$
r_{l}^{(p)}=r(t)-\sum_{\substack{j=1 \\ j \neq l}}^{L} \hat{I}_{j}^{(p-1)}\left(t-\tau_{j}\right)
$$

At the final iteration the soft output sequence of the MPIC is delivered to the turbo decoder to recover the transmitted information sequence.

\section{B. Soft symbol estimation}

Note $s=\left[s_{1}, s_{2}, \ldots, s_{M}\right]$ the modulated data sequence formed after the bit to symbol mapping of the data sequence $b=$ $\left[b_{1,1}, b_{1,2}, \ldots, b_{1, m}, \ldots, b_{i, 1}, b_{i, 2}, \ldots, b_{i, m}, \ldots, b_{M, 1}, b_{M, 2}, \ldots, b_{M, m}\right]$. $M$ is the number of modulated symbols, and $m=4$ in case of 16-QAM modulation. The LLR of the $i^{t h}$ bit of the $n^{\text {th }}$ symbol at $p^{\text {th }}$ iteration is expressed as

$$
\left.\tilde{b}_{k}^{(p)}(n, i)\right)=\ln \frac{\operatorname{Pr}\left(b_{k}(n, i)=+1 \mid z_{k}^{(p)}(n)\right)}{\operatorname{Pr}\left(b_{k}(n, i)=-1 \mid z_{k}^{(p)}(n)\right)}
$$

Note that the LLR of each bit is updated from iteration to iteration. This helps to generate a more accurate soft MPI replica. At $p^{\text {th }}$ iteration and for $k^{\text {th }}$ physical channel, the $n^{\text {th }}$ estimated soft modulation symbol, $\tilde{s}_{k}^{(p)}(n)$, is calculated by taking the mathematical expectation of the data symbol, $s_{k}(n)$, given the output of the SISO demapper. It is written as

$$
\begin{aligned}
\tilde{s}_{k}^{(p)}(n) & =E\left\{s_{k}(n) \mid \tilde{b}_{k}^{(p)}(n, 1), \ldots, \tilde{b}_{k}^{(p)}(n, 4)\right\} \\
& =\sum_{s_{k}(n) \in \mathcal{A}} s_{k}(n) \operatorname{Pr}\left(s_{k}(n) \mid \tilde{b}_{k}^{(p)}(n, 1), \ldots, \tilde{b}_{k}^{(p)}(n, 4)\right)
\end{aligned}
$$

where $\operatorname{Pr}($.$) is the probability. The estimated soft symbol,$ $\tilde{s}_{k}^{(p)}(n)$ can be rewriten as

$$
\tilde{s}_{k}^{(p)}(n)=\tilde{s}_{k, I}^{(p)}(n)+j \tilde{s}_{k, Q}^{(p)}(n),
$$

Where $\tilde{s}_{k, I}^{(p)}(n)$ and $\tilde{s}_{k, Q}^{(p)}(n)$ are the in-phase and quadraturephase components of $\tilde{s}_{k}^{(p)}(n)$ wich are expressed as

$$
\left\{\begin{array}{l}
\tilde{s}_{k, I}^{(p)}(n)=\frac{-1}{\sqrt{5}}\left[\left(\tanh \left(\tilde{b}_{k}^{(p)}(n, 1)\right)\right)\left(2+\tanh \left(\tilde{b}_{k}^{(p)}(n, 3)\right)\right)\right] \\
\tilde{s}_{k, Q}^{(p)}(n)=\frac{-1}{\sqrt{5}}\left[\left(\tanh \left(\tilde{b}_{k}^{(p)}(n, 2)\right)\right)\left(2+\tanh \left(\tilde{b}_{k}^{(p)}(n, 4)\right)\right)\right]
\end{array}\right.
$$

By substituting $\tilde{s}_{k}^{(p)}(n)$ into (3), we obtain the soft multipath interference replica.

\section{Soft ITERATIVE MPIC COMBINED WITH HARQ AND CONSTELLATION REARRANGEMENT}

\section{A. Soft iterative MPIC with HARQ}

The HSDPA introduced the HARQ technique as an other link adaptation in order to enhance the AMC performance. Fig. 2 shows the principle of HARQ with interference cancellation. The received packet is first processed by the iterative soft MPIC for interference cancellation. Then, it is delivered to the turbo decoder. A CRC detector is used for error detection. When the packet is erroneous, it is saved at the receiver and a NACK is sent to the transmetter which retransmits the packet. 


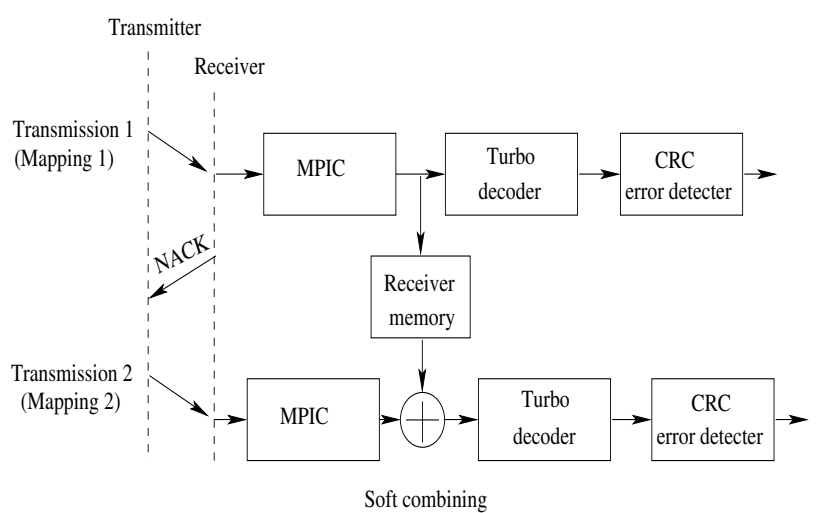

Fig. 2. Iterative soft MPIC combined with HARQ

At the receiver, the retransmitted packet is combined whith the orignal one before turbo decoding.

The most known of HARQ algorithms is Chase combining which is fist proposed in [9]. It was proven that Chase approach offers time diversity and provides a significant signal to noise ratio gain after retransmissions combining. In Chase combining, retransmissions are identical to the original transmission [11]. In addition to its reduced complexity, Chase combining requires a small memory.

\section{B. Constellation rearrangement}

In [13], it has been proven that there are variations in the bit reliabilities for 16-QAM modulation. These variations depend on the significance of the bit within a symbol: the two most significant bits are more relible than the two least significant bits [12], [13]. In case of Chase combining employing the same signal constellation mapping for all transmissions, the variations in bit reliabilities remain when soft-combining the received transmissions at bit level.

In this paper we propose an interworking of Chase combining and constellation rearrangement (CoRe) technique in order to combat the reliability variation problem. The constellation rearrangement averages the bit reliabilities by employing different signal mapping constellations for the HARQ transmissions (see Fig. 2). In this manner, some less protected bits becomes more protected. The bits within a 16-QAM symbol at the input of constellation rearrangement are denoted here by $i_{1} q_{1} i_{2} q_{2}$. The obtained signal constellation mappings are summarized in TABLE I [1]. The mapping \#1 represents the

TABLE I

CONSTELLATION REARRANGEMENT

\begin{tabular}{|c|c|c|}
\hline Mapping & Output bit sequence & High reliable bits \\
\hline Mapping \#1 & $i_{1} q_{1} i_{2} q_{2}$ & $i_{1} q_{1}$ \\
\hline Mapping \#2 & $i_{2} q_{2} i_{1} i_{2}$ & $i_{2} q_{2}$ \\
\hline Mapping \#3 & $i_{1} q_{2} \overline{i_{2} q_{2}}$ & $i_{1} q_{2}$ \\
\hline Mapping \#4 & $i_{2} q_{2} \overline{i_{1} q_{1}}$ & $i_{2} q_{2}$ \\
\hline
\end{tabular}

standard 16-QAM constellation (no rearrangement is made). The over-score on bits in TABLE I indicates that the logical values of this bits changes $(\overline{1}=0$ and $\overline{0}=1)$.

\section{Simulator CONFIgURATION}

For testing our scheme, we implemented the HSDPA link level simulator. It computes the resisuel BLER (Block Error Rate) and throughput for each SNR using monte carlo method. The throughput is given by this expression :

$$
\text { Throughput }=D .\left(1-B L E R_{(H A R Q)}\right),
$$

where $D$ is the information bit rate and $B L E R_{(H A R Q)}$ is the block error rate which takes into account Hybrid ARQ transmissions. At the transmitter, every function is performed according to 3GPP technical specifications [1], [2].

At the receiver, a Rake receiver using MRC (Maximum Ratio Combining) was applied to recover the transmitted signal. The iterative soft multpath interference canceller (MPIC) was implemented using soft interfernce estiamation and 4 iterations. It includes the soft output demapper for updating the soft bits values through MPIC iterations. The turbo decoding uses the Max-Log-MAP algorithm with 10 iterations. The channel model utilizes the profile of ITU-Pedestrian B channel with the speed of $3 \mathrm{Km} / \mathrm{h}$ (see Fig. 3). The receiver assumed a perfect channel knowledge. For HARQ, the Chase combining approach with constellation rearrangement technique was used. The maximum number of HARQ transmissions is fixed to 2. A delay of $12 \mathrm{~ms}$ is used between HARQ transmissions. The simulations were run using the radio link parameters summarized in TABLE II.



Fig. 3. The profile of ITU-Pedestrian B channel

TABLE II

SIMULATION PARAMETERS

\begin{tabular}{|c|c|}
\hline Chip rate & $3.84 \mathrm{Mcps}$ \\
\hline Symbol rate & $240 \mathrm{Ksps}$ \\
\hline Spreading Factor (SF) & 16 \\
\hline Transmission Time Interval (TTI) & $2 \mathrm{~ms}$ \\
\hline Power Control & None \\
\hline Spreading code & OVSF codes \\
\hline Modulation & $16-\mathrm{QAM}$ \\
\hline Channel coding/decoding & Turbo coding / Max-Log-MAP \\
\hline Number of turbo decoding iterations & 10 \\
\hline HARQ & Chase with CoRe \\
\hline CRC & 24 bits \\
\hline Max. Number of HARQ processes & 2 \\
\hline Delay between HARQ retransmissions (RTD) & $12 \mathrm{~ms}$ \\
\hline
\end{tabular}




\section{Simulation RESUlts}

Simulations were done for several channel quality indicator (CQI) modes to observe the performance enhancement. The predifined CQI modes used in this work are shown in TABLE III [2]. Performance is presented in terms of the residual BLER

TABLE III

MCSS CONFIGURATION

\begin{tabular}{|c|c|c|c|c|c|}
\hline CQI & Transport blocs size & Number of codes & Modulation & D (kbit/s) & R \\
\hline 16 & 3565 & 5 & 16-QAM & 1794.5 & 0.3739 \\
\hline 18 & 4688 & 5 & 16-QAM & 2344.0 & 0.4883 \\
\hline 22 & 7192 & 5 & 16-QAM & 3596.0 & 0.7492 \\
\hline 23 & 9743 & 7 & 16-QAM & 4871.5 & 0.7249 \\
\hline 24 & 11442 & 8 & 16-QAM & 5721.0 & 0.7449 \\
\hline
\end{tabular}

and throughput in function of the sum of code symbol signal to noise ratio which expressed as :

$$
S N R=10 \log _{10}\left(K \frac{E_{s}}{\sigma^{2}}\right)
$$

where $K$ is the number of codes (physical channels), $E_{s}$ is the symbol energy and $\sigma^{2}$ represents the noise variance.

BLER results of CQI_16, CQI_18 and CQI_22 are presented in Fig. 4, Fig. 5 and Fig. 6 respectively. We see that considerable parformance gain is offered by MPIC when combined with Chase combining. An additionnal performance gain is obtained with the constellation rearrangement which, in case of CQI_16 for example, achieved almost $2 \mathrm{~dB}$ gain over the technique using the interworking of MPIC and Chase combining without CoRe. For CQI_18, with the Rake, we



Fig. 4. BLER for CQI_16 in ITU-Pedestrian B channel

observe a performance floor which limited the BLER to a value above $10^{-1}$. With the MPIC and other techniques, the floor is completely removed. In case of CQI_22, the MPIC curve presents a performance floor due to the sever multipath interference caused by the ITU-Pedestrian B channel. This floor is suppressed when using HARQ. The use of CoRe with CoRe resulted in great performance enhancement over the MPIC. This is due to the diversities offered by Chase combining and CoRe.

BLER results for CQI_23 and CQI_24 are shown in Fig. 7 and Fig. 8 respectively. We see that for CQI_23 the error floor observed in MPIC curve, is removed by HARQ, whereas the HARQ curve for CQI_24 have a lowered floor compared

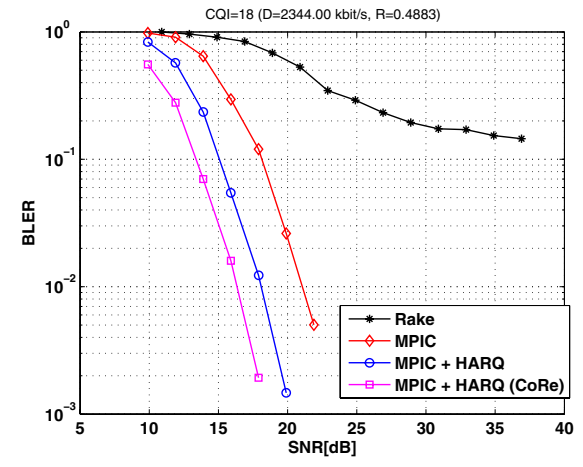

Fig. 5. BLER for CQI_18 in ITU-Pedestrian B channel

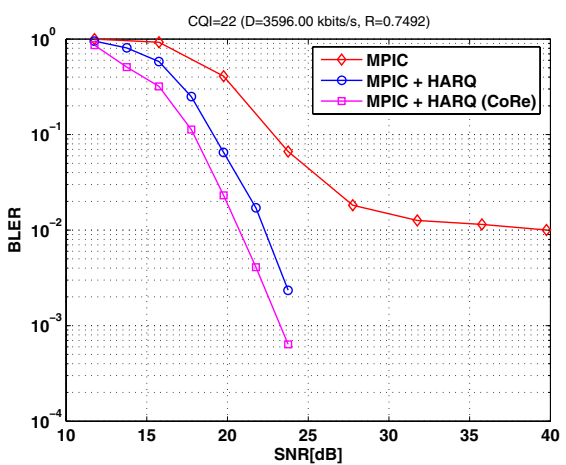

Fig. 6. BLER for CQI_22 in ITU-Pedestrian B channel

with the MPIC. This is due to the higher coding rate used by CQI_24 ( $\mathrm{R}=0.7449)$. It is also shown that best performance gain is offered by CoRe for both CQIs.

In order to see the impact of constellation rearrangement over

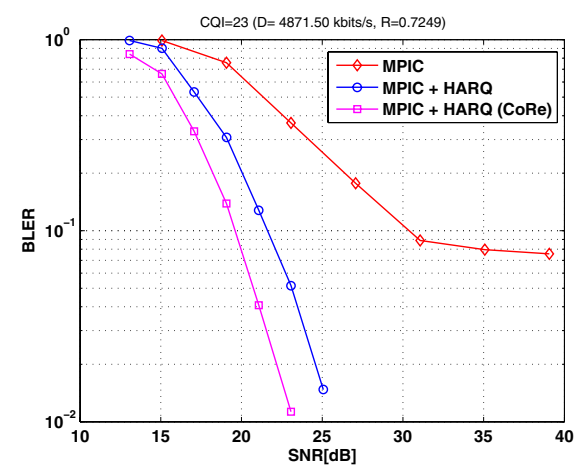

Fig. 7. BLER for CQI_23 in ITU-Pedestrian B channel

the throughput, simulations are done for CQI_16 and CQI_18. The throuput results of these CQIs are presented by Fig. 9 for CQI_16 and Fig. 10 for CQI_18. By analyzing these results, we observe that the throuput is signficantly improved at low signal to noise ratio.

\section{CONCLUSIONS}

In this paper, we have shown that the performance of HSDPA CQIs modes using 16-QAM modulation are not very 


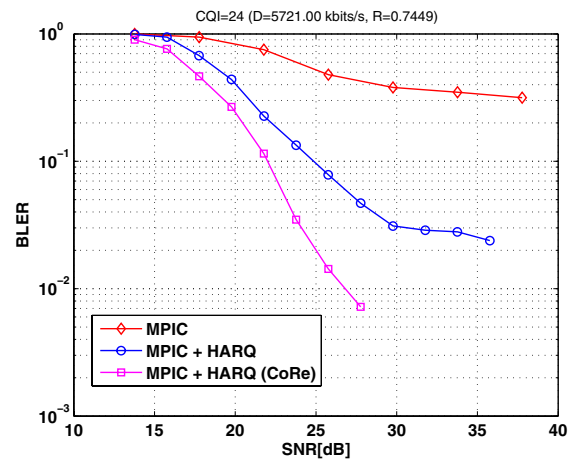

Fig. 8. BLER for CQI_24 in ITU-Pedestrian B channel



Fig. 9. Throughput for CQI_16 in ITU-Pedestrian B channel

well in multipath environment. This is due to high sensitivity of this modulation to interference. A soft iterative multipath interference canceller was used for suppressing this interference. It was shown that this intereference canceller resulted to a considerable performance enhancement. Besides, the use of HARQ technique and constellation rearrangement offered a great performance gain.

\section{REFERENCES}

[1] 3GPP TSG RAN, Multiplexing and channel coding (FDD), 3GPP TS 25.212 V6.2.0, Valbone,France, Jun. 2004.

[2] 3GPP TSG RAN, Spreading and modulation (FDD), 3GPP TS 25.213 V6.0.0, Valbone,France, Dec. 2003.

[3] K. Higuchi, A. Fujiwara and M. Sawahashi, Multipath Interference Canceller for High-Speed Packet Transmission with Adaptive Modulation and Coding Scheme in W-CDMA Forward Link, IEEE Journal on Commun., vol.20, February 2002, pp. 419-432.

[4] N. Miki, S. Abeta, H. Atarashi, and M. Sawahashi, Multipath Interference Canceller Using Soft-decision Replica Combined with Hybrid ARQ in W-CDMA Forward Link, IEEE VTC 2001-Fall, vol.3, 7-11 Oct. 2001, pp. $1922-1926$.

[5] J. C. Silva, N. Souto, A. Correia, F. Cercas and A. Rodrigues, Multipath interference canceller for high speed downlink packet access in enhanced UMTS networks, IEEE eighth International Symposium on Spread Spectrum Techniques and Applications, 30 Aug.-2 Sept. 2004, pp. 609 - 612

[6] T. Asai, K. Higuchi, M. Sawahashi, Field experiments on miltipath interference canceller associated with AMC in HSDPA, IEEE VTC 2003-Spring, vol.3, 22-25 April 2003, pp. 1624 - 1628.

[7] H. Yu, H. Cho, C. Kang and D. Hong, A New Multipath Interference Mitigation Technique for High-Speed Packet Transmission in WCDMA Downlink, IEEE Signal Processing Letters, vol.12, Issue9, Sept. 2005, pp. $601-604$.

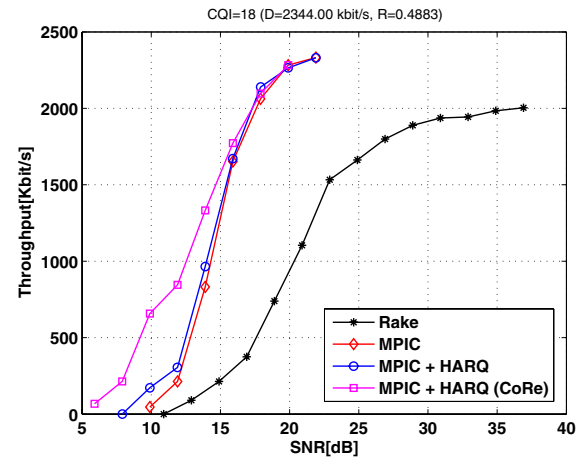

Fig. 10. Throughput for CQI_18 in ITU-Pedestrian B channel

[8] K. Ko, D. Kwon, D. Cho, C. Kang and D. Hong, Performance analysis of a multistage MPIC in 16-QAM CDMA systems over multipath Rayleigh fading channels, IEEE VTC 2003-Spring, vol.4, 22 - 25 April 2003, pp. $2807-2811$.

[9] D. Chase, Code Combining-A Maximum-Likelihood Decoding Approach for Combining an Arbitrary Number of Noisy Packets, IEEE Transactions on Communications, vol. 33, Issue 5, May 1985, pp. 385 - 395.

[10] M. Dottling, J. Michel and B. Raaf, Hybrid ARQ and adaptive modulation and coding schemes for high speed downlink packet access, IEEE PIMRC 2002, vol.3, 15-18 Sept. 2002, pp. 1073 - 1077.

[11] P. Frenger, S. Parkvall and E. Dahlman, Performance comparison of HARQ with Chase combining and incremental redundancy for HSDPA, IEEE VTC 2001, vol.3, 7-11 Oct. 2001, pp. 1829-1833.

[12] M. Dottling, T. Grundler and A. Seeger, Incremental redundancy and bit-mapping techniques for high speed downlink packet access, IEEE GLOBCOM 2003, vol.2, 1-5 Dec. 2003, pp. 908 - 912.

[13] N. Bliudze, N. Billy and D. Krob, On optimal hybrid ARQ control schemes for HSDPA with 16QAM, IEEE WiMob 2005, vol.1, 22-24 Aug. 2005, pp. $121-127$. 